\title{
COOPERATIVE STOCHASTIC CONTROL FOR OPTICAL BEAM TRACKING
}

\author{
ARASH KOMAEE \\ Department of Electrical and Computer Engineering \\ University of Maryland, College Park, MD 20742, USA
}

\begin{abstract}
Maintaining optical alignment between stations of a free-space optical link requires a persistent beam tracking operation. This is achieved using a position-sensitive photodetector at each station which measures the azimuth and elevation of tracking error. A pointing assembly adjusts the heading of transceivers according to measurement of the tracking error. The measurement at each receiver also depends on the pointing error of the opposite transmitter, therefore a cooperative beam tracking system can be regarded as two dynamically coupled subsystems. We developed a stochastic model for a cooperative beam tracking system to get insight into solution of an associated optimal control problem with goal of maximizing the flow of optical power between the stations.
\end{abstract}

\section{INTRODUCTION}

In free-space optical communication using narrow laser beams, it is required to maintain the alignment of transmitter and receiver stations in spite of their relative motion. This relative motion might be caused by the mobile nature of the stations, mechanical vibration, or accidental shocks. Prior to data transmission begins, coarse alignment is achieved through two operations: pointing and spatial acquisition. Pointing is the act of aiming the transmitted beam toward the receiver within an acceptable accuracy. The purpose of spatial acquisition is to detect the transmitter's beam and align the normal vector to the receiving optical device with the direction of the impinging optical field. Following a coarse alignment accomplished in the acquisition phase, data transmission is established and simultaneously the operation of cooperative beam tracking is performed. This fine alignment operation is intended to precisely compensate for persistent relative motion of the stations. For detailed description of these three operations we refer the reader to [1, 2]. In this study we are concerned about cooperative beam tracking.

In a cooperative beam tracking system, the stations continuously track the arrival direction of the incident beam and transmit their beam back in that direction. A positionsensitive photodetector (e.g. quadrant detector) in association with a focusing lens is employed at each station to measure the azimuth and elevation components of the error vector.

This research was supported by the Army Research Office under ODDR\&E MURI01 Program Grant No. DAAD19-01-1-0465 to the Center for Communicating Networked Control Systems (through Boston University), and by the National Science Foundation under Grant No. ECS 0636613.

E-mail address: akomaee@eng.umd.edu. 
The error vector is the displacement of the beam's arrival direction with respect to the direction normal to the receiving aperture. A servo-driven pointing assembly adjusts the heading of the transmitting optical device (in azimuth and elevation directions) according to the measured error vector. Normally, a single pointing assembly is used to control the direction of both receiving and transmitting optics, which are installed on the same platform.

Cooperative tracking systems were already studied by other researchers based on deterministic models [1, 3, 4]. These models describe the position-sensitive photodetector by a deterministic input-output relationship and employ deterministic functions to characterize the relative motion of the stations. Using such a deterministic model, under a proportional control law, Wei and Gagliardi [3] evaluated the steady-state performance of the system and Marola et al. studied stability properties [4].

For most applications, a stochastic model may be more appropriate for relative motion of the stations. Moreover, the optical sensing devices are usually described by stochastic models [1, 5]. This leads us to suggest that a stochastic approach is more likely to lead to rigorous analysis and design of cooperative tracking systems. In this paper, we develop a stochastic model for cooperative tracking systems and employ the model to analyze the system and study an associated optimal control problem. Maximizing the flow of the optical power between the stations shall be our criterion for optimality.

\section{System Description}

We consider an optical transceiver comprised of a lens, a position-sensitive photodetector, and a narrow laser source, all installed on a rigid platform. The photodetector surface is perpendicular to the lens axis and its center is placed at the focus of the lens. The axes of the lens and the laser source are parallel to the transceiver axis. The azimuth and elevation of the transceiver axis can be controlled by means of a servo-driven pointing assembly. A two-way optical link employs two transceivers of this type in such a manner that each transceiver transmits its optical beam toward the opposite station and receives the optical beam from the opposite side. The optical beams are used for two purposes: as a carrier of information and as a beacon assisting the opposite station in its tracking operation. We assume that the stations are subjected to relative motion.

In what follows, we distinguish the stations by superscripts $a$ and $b$ or $i=a, b$ when referring to both stations. The dependence on time will be shown by subscript $t$. The twodimensional vector $\theta_{t}^{i}$ denotes the azimuth and elevation angles of the transceiver axis $i$ with respect to some fixed coordinate system. Similarly, $\phi_{t}$ denotes the azimuth and elevation angles of the line-of-sight (LOS) of the stations with respect to same coordinate system. We define the tracking error of station $i$ as $\psi_{t}^{i}=\theta_{t}^{i}-\phi_{t}$ which is equivalent to the pointing error for the opposite station.

We shall assume that the transmitted optical fields impinge the receivers along the lineof-sight of the stations, regardless of the pointing error of the transmitters. This implies that the received optical field at station $i$ strikes the receiving lens along the error vector $\psi_{t}^{i}$ with respect to the axis of the lens. The validity of this assumption is clear for a spherical optical field. For a Gaussian beam [6, which is the model used in this study, we can show that the angle between LOS and the beam arrival direction at the receiver depends on the third 
power of the pointing error at the transmitter. Because the pointing error is maintained small by means of feedback control, its third power can be neglected at least for a linear model, which justifies our assumption.

In contrast to the beam arrival direction, the intensity of the received optical field substantially depends on the transmitter's pointing error. Assume that station $b$ transmits a circular symmetric Gaussian beam with divergence angle $2 \bar{\psi}$ toward station $a$. Then, due to the pointing error $\psi_{t}^{b}$ at station $b$, the instantaneous optical power received by station $a$ at time $t$ is reduced by a factor of $\exp \left(-2\left\|\psi_{t}^{b}\right\|^{2} / \bar{\psi}^{2}\right)$. This attenuation factor is obtained based on two assumption: first, the radius of the optical beam is much larger than the receiver aperture, and second, the distance between the stations is short enough to allow ignoring the propagation delay.

The image of the received optical field over the surface of the photodetector is a spot of light with a bell-shaped intensity profile centered at $y_{t}^{i}=f_{c} \psi_{t}^{i}$, where $f_{c}$ is the focal length of the lens [1]. Let $\Omega(r)$ be the intensity pattern of the spot of light, where $r$ is the position vector of a point on the surface of the photodetector. Denote by $P_{t}^{a}>0$ the total optical power received by station $a$ at time $t$ in the absence of pointing error $\psi_{t}^{b}$. Then the optical intensity over the surface of photodetector $a$ is given by

$$
I_{t}^{a}(r)=P_{t}^{a} \exp \left(-2\left\|\psi_{t}^{b}\right\|^{2} / \bar{\psi}^{2}\right) \Omega\left(r-f_{c} \psi_{t}^{a}\right)
$$

A similar expression can be obtained for $I_{t}^{b}(r)$ by flipping $a$ and $b$ in (1). Note that the displacement of the spot of light depends only on the error vector of the same station, while the total received power depends on the error vector of the opposite station.

Since $\psi_{t}^{i}$ depends linearly on the displacement of the spot of light, it can be estimated from the output of the position-sensitive photodetector. This estimate is provided to a controller which applies proper control signals to the pointing assembly in order to drive the error vector to zero. Note that $\psi_{t}^{a}=\psi_{t}^{b}=0$ is the "unattainable" goal of a cooperative tracking system. Under a realistic condition, the objective of the system is to maintain $\left\|\psi_{t}^{a}\right\|$ and $\left\|\psi_{t}^{b}\right\|$ as small as possible. Since the axis of the laser source is parallel to the transceiver's axis, $\psi_{t}^{i}=0$ implies that the station $i$ transmits its optical beam along LOS, which leads to maximum flow of the optical power between the stations.

From the above description, we find out that a cooperative tracking system consists of two dynamical subsystems coupled via their measurement. The subsystems operate cooperatively in the sense that a small pointing error at one station assists the other station by increasing its received optical power which leads to more accurate estimation of its error vector.

\section{The Model}

The model we use in this study is a two-station extension of the single-station model in [7. We refer the reader to that article for detailed description and justification of the model. Without loss of generality, we assume the stations are identical.

The pointing assembly is an electro-mechanical system with the input vector $u_{t}^{i} \in \mathbb{R}^{2}$ and the output vector $\theta_{t}^{i} \in \mathbb{R}^{2}$. The input and output vectors are two-dimensional corresponding to the azimuth and elevation angles. We model this system by the linear stochastic 
differential equation

$$
\begin{aligned}
d x_{t}^{p, i} & =A_{t}^{p} x_{t}^{p, i} d t+B_{t}^{p} u_{t}^{i} d t+D_{t}^{p} d w_{t}^{p, i} \\
\theta_{t}^{i} & =C_{t}^{p} x_{t}^{p, i}
\end{aligned}
$$

where $x_{t}^{p, i} \in \mathbb{R}^{n_{p}}$ is the state vector, $\left\{w_{t}^{p, i}, t \geqslant 0\right\}$ is a $m_{p}$-dimensional standard Wiener process, and $A_{t}^{p}, B_{t}^{p}, D_{t}^{p}$, and $C_{t}^{p}$ are uniformly bounded matrices with proper dimensions. We assume that $\left\{w_{t}^{p, a}, t \geqslant 0\right\}$ and $\left\{w_{t}^{p, b}, t \geqslant 0\right\}$ are mutually independent.

Using a linear model for the pointing assembly is justified by the fact that the system operates over small angles during the fine control regime. In applications like intersatellite communication, the relative motion consists of a large, deterministic component and a small, stochastic term. Accordingly, the control law consists of a deterministic, open-loop, coarse control and a small, closed-loop, fine control. In this case, the nonlinear state equations describing the system is linearized around the deterministic nominal trajectory, which results in the time-varying model (2) for the fine control regime.

We model $\phi_{t}$ by a Gauss-Markov stochastic process described by the state-space equations

$$
\begin{aligned}
d x_{t}^{d} & =A_{t}^{d} x_{t}^{d} d t+D_{t}^{d} d w_{t}^{d} \\
\phi_{t} & =C_{t}^{d} x_{t}^{d}
\end{aligned}
$$

with state vector $x_{t}^{d} \in \mathbb{R}^{n_{d}}, m_{d}$-dimensional standard Wiener process $\left\{w_{t}^{d}, t \geqslant 0\right\}$, and uniformly bounded matrices $A_{t}^{d}, D_{t}^{d}$, and $C_{t}^{d}$ with proper dimensions.

The position vector $y_{t}^{i}=f_{c} \psi_{t}^{i}$ is a linear function of $x_{t}^{p, i}$ and $x_{t}^{d}$, so we can combine equations (2) and (3) and write them in the compact form

$$
\begin{aligned}
d x_{t}^{i} & =A_{t} x_{t}^{i} d t+B_{t} u_{t}^{i} d t+D_{t} d w_{t}^{i} \\
y_{t}^{i} & =C_{t} x_{t}^{i}
\end{aligned}
$$

with state vector $x_{t}^{i} \in \mathbb{R}^{n}$ and $m$-dimensional standard Wiener process $\left\{w_{t}^{i}, t \geqslant 0\right\}$, where $n=$ $n_{p}+n_{d}$ and $m=m_{p}+m_{d}$. The initial state $x_{0}^{i}$ is assumed to be a Gaussian vector with mean $\bar{x}_{0}^{i}$ and covariance matrix $\bar{\Sigma}_{0}^{i}$ and independent of $\left\{w_{t}^{a}, t \geqslant 0\right\}$ and $\left\{w_{t}^{b}, t \geqslant 0\right\}$.

We approximate the bell-shaped intensity profile $\Omega(r)$ in (1) by a Gaussian function. Then, in terms of the state vectors $x_{t}^{a}$ and $x_{t}^{b}$, the optical intensity $I_{t}^{a}(r)$ can be expressed as

$$
I_{t}^{a}(r)=P_{t}^{a} \exp \left(-\rho\left\|C_{t} x_{t}^{b}\right\|^{2}\right) \gamma_{t}\left(r, x_{t}^{a}\right)
$$

where $\rho=2 /\left(\bar{\psi} f_{c}\right)^{2}$ and $\gamma_{t}(r, x)$ is defined as

$$
\gamma_{t}(r, x)=(2 \pi)^{-1}\left(\operatorname{det} R_{t}\right)^{-1 / 2} \exp \left\{-\frac{1}{2}\left(r-C_{t} x\right)^{T}\left(R_{t}\right)^{-1}\left(r-C_{t} x\right)\right\}
$$

Here, $R_{t}=R_{t}^{T}$ is a $2 \times 2$ positive-definite matrix describing the shape of the pattern. For a circular symmetric pattern with constant radius $\varrho>0$ we have $R_{t}=\varrho I_{2 \times 2}$. A similar expression can be derived for $I_{t}^{b}(r)$ by exchanging $a$ and $b$. We remind that in (5) the propagation delay is neglected.

We allow $P_{t}^{a}$ and $P_{t}^{b}$ to be nonnegative stochastic processes with piecewise continuous sample paths and nonzero expectations to model the random optical fade caused by atmospheric turbulence and aerosols and the information-bearing signals modulating the optical 
beams. Further, we assume that $\left\{P_{t}^{a}, t \geqslant 0\right\}$ and $\left\{P_{t}^{b}, t \geqslant 0\right\}$ are mutually independent and independent of $x_{0}^{i}$ and $\left\{w_{t}^{i}, t \geqslant 0\right\}, i=a, b$.

The position-sensitive photodetector is a photoelectron converter whose surface is partitioned into small regions. The output of each region counts the number of converted electrons regardless of their location on the region. The photoelectron conversion rate depends linearly on the optical power absorbed by the region. Generally, a photoelectron converter is modeled by a Poisson process with a rate proportional to the impinging optical power [1, 5]. In the present case, where the optical power is a stochastic field, the output of each region shall be modeled by a conditionally (doubly stochastic) Poisson process.

Many practical beam tracking systems employ a quadrant detector, a photodetector with a four-region partition, as their optical sensing device. However, the low spatial resolution of the quadrant detector can be improved using a finer partition. For instance, the authors of [8] describe a beam tracking system utilizing a photodetector with $512 \times 512$ pixels. In this study, following [7], we use an infinite resolution model for the photodetector. This idealized model provides a reasonable approximation for high resolution photodetectors. We also make another ideal assumption that the surface of the photodetector is infinitely large [7]. This assumption is justified when the photodetector area is significantly larger than the size and the displacement of the spot of light. We believe that the control law obtained from this idealized model provides a useful point of departure for practical designs, even for low resolution or finite area photodetectors.

We use a doubly stochastic space-time Poisson process to describe the output of an infinite resolution photodetector [7. The rate of this process is assumed to be proportional to $I_{t}^{i}(r)$. From (5), the stochastic rate associated to photodetector $i$ can be expressed as

$$
\lambda_{t}\left(r, x_{t}^{i}, \mu_{t}^{i}\right)=\mu_{t}^{i} \gamma_{t}\left(r, x_{t}^{i}\right)
$$

where $\mu_{t}^{a}$ and $\mu_{t}^{b}$ are defined as

$$
\begin{aligned}
& \mu_{t}^{a}=\nu_{t}^{a} \exp \left(-\rho\left\|C_{t} x_{t}^{b}\right\|^{2}\right) \\
& \mu_{t}^{b}=\nu_{t}^{b} \exp \left(-\rho\left\|C_{t} x_{t}^{a}\right\|^{2}\right)
\end{aligned}
$$

Here, $\nu_{t}^{i}$ is defined as $\nu_{t}^{i}=\eta P_{t}^{i}$, where $\eta>0$ is the sensitivity of the photodetectors and is assumed to be a constant. Note that $\nu_{t}^{i}$ inherits the statistical properties of $P_{t}^{i}$.

The space-time Poisson process, defined over $[0, \infty) \times \mathbb{R}^{2}$, characterizes the occurrence of discrete events (e.g. release of a single electron) with a temporal component $t \in[0, \infty)$ and a spatial component $r \in \mathbb{R}^{2}$. Let $\mathcal{T}$ and $\mathcal{S}$ be Borel sets in $[0, \infty)$ and $\mathbb{R}^{2}$ respectively and $N^{i}(\mathcal{T} \times \mathcal{S})$ denote the number of points occurring in $\mathcal{T} \times \mathcal{S}$. Define the random variable

$$
\Lambda^{i}(\mathcal{T} \times \mathcal{S})=\int_{\mathcal{T} \times \mathcal{S}} \lambda_{t}\left(r, x_{t}^{i}, \mu_{t}^{i}\right) d t d r
$$

Then $N^{i}(\mathcal{T} \times \mathcal{S})$ is a doubly stochastic Poisson random variable with conditional probability distribution

$$
\operatorname{Pr}\left\{N^{i}(\mathcal{T} \times \mathcal{S})=n \mid \Lambda^{i}(\mathcal{T} \times \mathcal{S})\right\}=\frac{e^{-\Lambda^{i}(\mathcal{T} \times \mathcal{S})}\left(\Lambda^{i}(\mathcal{T} \times \mathcal{S})\right)^{n}}{n !}
$$

Moreover, for disjoint $\mathcal{T}_{1} \times \mathcal{S}_{1}$ and $\mathcal{T}_{2} \times \mathcal{S}_{2}$, conditioned on $\Lambda^{i}\left(\mathcal{T}_{1} \times \mathcal{S}_{1}\right)$ and $\Lambda^{j}\left(\mathcal{T}_{2} \times \mathcal{S}_{2}\right)$, the random variables $N^{i}\left(\mathcal{T}_{1} \times \mathcal{S}_{1}\right)$ and $N^{j}\left(\mathcal{T}_{2} \times \mathcal{S}_{2}\right)$ are independent for $i=a, b$ and $j=a, b$. 
Let $(\Omega, \mathscr{F}, P)$ be the underlying probability space for the above stochastic model. Define $\mathscr{B}_{t}^{i}$ as the $\sigma$-algebra generated by the space-time process $i$ over $[0, t)$. We define the counting process $N_{t}^{i}$ as the number of points occurred during $[0, t)$ over the entire surface of photodetector $i$ regardless of their location, i.e., $N_{t}^{i}=N^{i}\left([0, t) \times \mathbb{R}^{2}\right)$. We say $u_{t}^{a}$ and $u_{t}^{b}$ are admissible controls if $u_{t}^{i}$ is $\mathscr{B}_{t}^{i}$-measurable and the solution to (44) is well defined for $i=a, b$.

Let $T$ be an arbitrary positive constant. We define the objective functional

$$
J=\mathrm{E}\left[\int_{0}^{T}\left(\alpha^{a} \nu_{t}^{a} \exp \left(-\rho\left\|C_{t} x_{t}^{b}\right\|^{2}\right)+\alpha^{b} \nu_{t}^{b} \exp \left(-\rho\left\|C_{t} x_{t}^{a}\right\|^{2}\right)\right) d t\right]
$$

where $\alpha^{i} \geqslant 0, i=a, b$. Evidently, $J$ is a linear combination of the expected optical energy received by the stations during $[0, T]$. Our goal is to obtain admissible controls $u_{t}^{a}$ and $u_{t}^{b}$ that maximize $J$.

\section{The Control Problem}

We first outline some results in the estimation of the state vector $x_{t}^{i}$ due to Rhodes and Snyder [9]. Later, we will utilize these results to approach the control problem. Before moving forward, let us fix some notation. Let $\left(t_{k-1}, t_{k}\right]$ be the interval between two successive occurrence of the space-time process and $r_{k}$ be the location of $k^{t h}$ occurring point. Assume that $h_{t}\left(r, \xi_{t}\right)$ is continuous in $r$ and left continuous in $t$ and $\xi_{t}$. Then the stochastic differential equation

$$
d \xi_{t}=\int_{\mathbb{R}^{2}} h_{t}\left(r, \xi_{t}\right) N(d t \times d r)
$$

is defined such that $d \xi_{t}=0$ during $\left(t_{k-1}, t_{k}\right]$ and $\xi_{t}$ encounters a jump of $h_{t_{k}}\left(r_{k}, \xi_{t_{k}}\right)$ at $t=t_{k}$.

Consider the state-space model (44) and its associated space-time observation with the rate process (7). Assume that we are given the increasing family of $\sigma$-algebras $\mathscr{B}_{t}^{i}$ and $u_{t}^{i}$ is $\mathscr{B}_{t}^{i}$-measurable. Then, regardless of the nature of $\mu_{t}^{i}$, the posterior density $p_{x_{t}^{i}}\left(x \mid \mathscr{B}_{t}^{i}\right)$ is Gaussian with mean $\hat{x}_{t}^{i}$ and covariance matrix $\Sigma_{t}^{i}$ determined from the stochastic differential equations [9]

$$
\begin{aligned}
& d \hat{x}_{t}^{i}=A_{t} \hat{x}_{t}^{i} d t+B_{t} u_{t}^{i} d t+\int_{\mathbb{R}^{2}} M_{t}^{i}\left(r-C_{t} \hat{x}_{t}^{i}\right) N^{i}(d t \times d r) \\
& d \Sigma_{t}^{i}=A_{t} \Sigma_{t}^{i} d t+\Sigma_{t}^{i} A_{t}^{T} d t+D_{t} D_{t}^{T} d t-M_{t}^{i} C_{t} \Sigma_{t}^{i} d N_{t}^{i}
\end{aligned}
$$

with initial states $\hat{x}_{0}^{i}=\bar{x}_{0}^{i}$ and $\Sigma_{0}^{i}=\bar{\Sigma}_{0}^{i}$, where $M_{t}^{i}$ is defined as

$$
M_{t}^{i}=\Sigma_{t}^{i} C_{t}^{T}\left(C_{t} \Sigma_{t}^{i} C_{t}^{T}+R_{t}\right)^{-1}
$$

Moreover, the conditional covariance matrix $\Sigma_{t}^{i}$ obtained from (11) is almost surely positive definite for $t>0$, provided that $\bar{\Sigma}_{0}^{i}$ is positive definite.

Note that the formulas (10) and (11) not explicitly depend on $\left\{\mu_{t}^{i}, t \geqslant 0\right\}$, however, the estimates $\hat{x}_{t}^{i}$ and $\Sigma_{t}^{i}$ depend on $\left\{\mu_{t}^{i}, t \geqslant 0\right\}$ through the observation $N^{i}(d t \times d r)$. This dependence can be explained by observing from (11) that the occurrence of each event in the space-time process subtracts the positive definite matrix $M_{t}^{i} C_{t} \Sigma_{t}^{i}$ from $\Sigma_{t}^{i}$, thus a larger $\mu_{t}^{i}$ leads to a smaller estimation error covariance by increasing the occurrence rate of the events. According to (8), a smaller pointing error $\left\|C_{t} x_{t}^{b}\right\|$ at station $b$ results in a 
larger $\mu_{t}^{a}$ and, as a consequence, a closer estimation for $x_{t}^{a}$, which in turn, leads to a smaller pointing error at station $a$. This explains the mechanism which couples the dynamics of the stations.

We exploit the above results to prove theorem 1 below which determines an upper bound on $J$ and establishes the conditions on $\hat{x}_{t}^{a}$ and $\hat{x}_{t}^{b}$ under which the upper bound can be achieved. Before stating the theorem, we fix notation. Let $\Sigma=\left[\sigma_{i j}\right]$ denote a symmetric $n \times n$ matrix and $f(\Sigma)$ be a scalar function of $\Sigma$. Assume that the partial derivatives of $f(\Sigma)$ with respect to elements of $\Sigma$ exist. We denote by $\partial f(\Sigma) / \partial \Sigma$ a $n \times n$ symmetric matrix $F(\Sigma)=\left[F_{i j}(\Sigma)\right]$ such that $F_{i i}=\partial f / \partial \sigma_{i i}$ and $F_{i j}=(1 / 2) \partial f / \partial \sigma_{i j}$ for $i \neq j$. Let $g_{t}\left(\Sigma^{a}, \Sigma^{b}\right)$ be a function of $n \times n$ symmetric matrices $\Sigma^{a}$ and $\Sigma^{b}$ with values in $\mathbb{R}$. We define the linear operators $\mathcal{L}_{t}^{a}\{\cdot\}$ and $\mathcal{L}_{t}^{b}\{\cdot\}$ as

$$
\begin{aligned}
& \mathcal{L}_{t}^{a}\left\{g_{t}\left(\Sigma^{a}, \Sigma^{b}\right)\right\}=g_{t}\left(S_{t}\left(\Sigma^{a}\right), \Sigma^{b}\right)-g_{t}\left(\Sigma^{a}, \Sigma^{b}\right) \\
& \mathcal{L}_{t}^{b}\left\{g_{t}\left(\Sigma^{a}, \Sigma^{b}\right)\right\}=g_{t}\left(\Sigma^{a}, S_{t}\left(\Sigma^{b}\right)\right)-g_{t}\left(\Sigma^{a}, \Sigma^{b}\right)
\end{aligned}
$$

where $S_{t}(\cdot)$ is defined as

$$
S_{t}(\Sigma)=\Sigma-\Sigma C_{t}^{T}\left(C_{t} \Sigma C_{t}^{T}+R_{t}\right)^{-1} C_{t} \Sigma
$$

With $I$ being the $2 \times 2$ identity matrix, we define the $2 \times 2$ positive definite matrix $Q_{t}^{i}$ as

$$
Q_{t}^{i}=\left(I+2 \rho C_{t} \Sigma_{t}^{i} C_{t}^{T}\right)^{-1 / 2}
$$

Also we define $q_{t}^{i}>0$ as

$$
q_{t}^{i}=\operatorname{det}\left(Q_{t}^{i}\right)
$$

Theorem 1. Fix sample paths $\nu_{t}^{a}$ and $\nu_{t}^{b}, t \in[0, T]$. Let $\Sigma^{a}$ and $\Sigma^{b}$ be $n \times n$ symmetric matrices. Assume that $g_{t}\left(\Sigma^{a}, \Sigma^{b}\right), t \in[0, T]$ is the backward solution to the partial differential equation

$$
\begin{aligned}
-\frac{\partial g_{t}\left(\Sigma^{a}, \Sigma^{b}\right)}{\partial t} & =\nu_{t}^{a} \frac{\alpha^{a}+\mathcal{L}_{t}^{a}\left\{g_{t}\left(\Sigma^{a}, \Sigma^{b}\right)\right\}}{\sqrt{\operatorname{det}\left(I+2 \rho C_{t} \Sigma^{b} C_{t}^{T}\right)}}+\nu_{t}^{b} \frac{\alpha^{b}+\mathcal{L}_{t}^{b}\left\{g_{t}\left(\Sigma^{a}, \Sigma^{b}\right)\right\}}{\sqrt{\operatorname{det}\left(I+2 \rho C_{t} \Sigma^{a} C_{t}^{T}\right)}} \\
& +\operatorname{tr}\left\{\frac{\partial g_{t}\left(\Sigma^{a}, \Sigma^{b}\right)}{\partial \Sigma^{a}}\left(A_{t} \Sigma^{a}+\Sigma^{a} A_{t}^{T}+D_{t} D_{t}^{T}\right)\right\} \\
& +\operatorname{tr}\left\{\frac{\partial g_{t}\left(\Sigma^{a}, \Sigma^{b}\right)}{\partial \Sigma^{b}}\left(A_{t} \Sigma^{b}+\Sigma^{b} A_{t}^{T}+D_{t} D_{t}^{T}\right)\right\}
\end{aligned}
$$

with boundary condition $g_{T}(\cdot, \cdot)=0$. Then for fixed sample paths of $\nu_{t}^{a}$ and $\nu_{t}^{b}$, the objective functional (9) can be expressed as

$$
\begin{array}{r}
J=g_{0}\left(\Sigma_{0}^{a}, \Sigma_{0}^{b}\right)-\mathrm{E}\left[\int_{0}^{T} \nu_{t}^{a} q_{t}^{b}\left(\alpha^{a}+\mathcal{L}_{t}^{a}\left\{g_{t}\left(\Sigma_{t}^{a}, \Sigma_{t}^{b}\right)\right\}\right)\left\{1-\exp \left(-\rho\left\|Q_{t}^{b} C_{t} \hat{x}_{t}^{b}\right\|^{2}\right)\right\} d t\right] \\
-\mathrm{E}\left[\int_{0}^{T} \nu_{t}^{b} q_{t}^{a}\left(\alpha^{b}+\mathcal{L}_{t}^{b}\left\{g_{t}\left(\Sigma_{t}^{a}, \Sigma_{t}^{b}\right)\right\}\right)\left\{1-\exp \left(-\rho\left\|Q_{t}^{a} C_{t} \hat{x}_{t}^{a}\right\|^{2}\right)\right\} d t\right]
\end{array}
$$

Moreover, if $\Sigma^{a}$ and $\Sigma^{b}$ are positive definite, $\mathcal{L}_{t}^{a}\left\{g_{t}\left(\Sigma^{a}, \Sigma^{b}\right)\right\}$ and $\mathcal{L}_{t}^{b}\left\{g_{t}\left(\Sigma^{a}, \Sigma^{b}\right)\right\}$ are positive for $t \in[0, T)$, provided that $C_{t}$ is full rank for any $t \in[0, T)$. 
Proof. See appendix A.

Corollary 1. For fixed sample paths $\nu_{t}^{a}$ and $\nu_{t}^{b}$ and any choice of positive definite matrices $\Sigma_{0}^{a}$ and $\Sigma_{0}^{b}, J^{*} \triangleq g_{0}\left(\Sigma_{0}^{a}, \Sigma_{0}^{b}\right)$ is an upper bound for $J$, i.e., $J \leqslant J^{*}$, and equality holds if and only if $C_{t} \hat{x}_{t}^{a}=0$ for $t \in \mathscr{T}^{a}$ and $C_{t} \hat{x}_{t}^{b}=0$ for $t \in \mathscr{T}^{b}$ almost everywhere. Here $\mathscr{T}^{a}$ and $\mathscr{T}^{b}$ are defined for fixed sample paths $\nu_{t}^{a}$ and $\nu_{t}^{b}$ as $\mathscr{T}^{a}=\left\{t \mid \nu_{t}^{b} \neq 0, t \in[0, T)\right\}$ and $\mathscr{T}^{b}=\left\{t \mid \nu_{t}^{a} \neq 0, t \in[0, T)\right\}$.

Proof. The second statement of theorem 1 in conjunction with positive definiteness of $\Sigma_{t}^{i}, i=$ $a, b$ results $\mathcal{L}_{t}^{i}\left\{g_{t}\left(\Sigma_{t}^{a}, \Sigma_{t}^{b}\right)\right\}>0$ for $i=a, b$ and $t \in[0, T)$. It follows that the integrands in the first and second integrals of (17) are positive over $\mathscr{T}^{b}$ and $\mathscr{T}^{a}$, respectively, except when $C_{t} \hat{x}_{t}^{b}=0$ for $t \in \mathscr{T}^{b}$ and $C_{t} \hat{x}_{t}^{a}=0$ for $t \in \mathscr{T}^{a}$, in which they are equal to zero. This leads to $J-J^{*} \leqslant 0$ with equality when the integrals vanish. The last condition holds if and only if $C_{t} \hat{x}_{t}^{b}=0$ for $t \in \mathscr{T}^{b}$ and $C_{t} \hat{x}_{t}^{a}=0$ for $t \in \mathscr{T}^{a}$ almost everywhere.

Corollary 2. For nonnegative stochastic processes $\nu_{t}^{a}$ and $\nu_{t}^{b}$ with piecewise continuous sample paths and nonzero expectations, the objective functional (9) achieves its maximum if and only if

$$
C_{t} \hat{x}_{t}^{a}=C_{t} \hat{x}_{t}^{b}=0
$$

for $t \in[0, T)$ almost everywhere.

Proof. If (18) holds, corollary 1 implies that for any sample path of $\left(\nu_{t}^{a}, \nu_{t}^{b}\right)$ the objective functional $J$ associated to that sample path meets its upper bound. This suggests that (18) is a sufficient condition for $J$ to achieve its maximum. To show that (18) is a necessary condition, assume that for some interval $\mathscr{I} \subseteq[0, T), C_{t} \hat{x}_{t}^{a} \neq 0$ or $C_{t} \hat{x}_{t}^{b} \neq 0$. Because $\nu_{t}^{a}$ and $\nu_{t}^{b}$ have nonzero expectations, with nonzero probability some of their sample paths are positive over $\mathscr{I}$. Then, corollary 1 implies that for those sample paths the condition for achieving the maximum is not satisfied. Therefore, the objective functional $J$ cannot achieve its maximum.

Remark 1. According to (10) and (11), $\hat{x}_{t}^{a}$ and $\hat{x}_{t}^{b}$ do not explicitly depend on $\nu_{t}^{a}$ and $\nu_{t}^{b}$. This suggests that the optimal control law not explicitly depends on $\nu_{t}^{a}$ and $\nu_{t}^{b}$.

Remark 2. The condition which leads to the upper bound $J^{*}$ does not depend on $\alpha^{a}$ and $\alpha^{b}$. In particular, the condition is same for $\left(\alpha^{a}, \alpha^{b}\right)=(1,0)$ and $\left(\alpha^{a}, \alpha^{b}\right)=(0,1)$. This means that if $C_{t} \hat{x}_{t}^{a}=C_{t} \hat{x}_{t}^{b}=0$ holds during $t \in[0, T)$, both stations receive the maximum possible optical energy.

The following lemma proposes a control law which leads to the condition $C_{t} \hat{x}_{t}^{a}=C_{t} \hat{x}_{t}^{b}=0$.

Lemma 1. Consider the stochastic dynamical system (10) and assume that $C_{0} \hat{x}_{0}^{i}=0$. Let $C_{t} B_{t}$ be nonsingular and $C_{t}$ be differentiable for $t \geqslant 0$. Then under control

$$
u_{t}^{i} d t=-\left(C_{t} B_{t}\right)^{-1}\left\{\left(C_{t} A_{t}+\dot{C}_{t}\right) \hat{x}_{t}^{i} d t+C_{t} M_{t}^{i} \int_{\mathbb{R}^{2}} r N^{i}(d t \times d r)\right\}
$$

we have $C_{t} \hat{x}_{t}^{i}=0$ for any $t \geqslant 0$. 
Proof. We verify the validity of the lemma by putting

$$
u_{t}^{i} d t=-\left(C_{t} B_{t}\right)^{-1}\left\{\left(C_{t} A_{t}+\dot{C}_{t}\right) \hat{x}_{t}^{i} d t+\int_{\mathbb{R}^{2}} C_{t} M_{t}^{i}\left(r-C_{t} \hat{x}_{t}^{i}\right) N^{i}(d t \times d r)\right\}
$$

into (10) and left multiplying both sides by $C_{t}$. The resulting equation will be $C_{t} d \hat{x}_{t}^{i}=$ $-\dot{C}_{t} \hat{x}_{t}^{i} d t$, which yields $d\left(C_{t} \hat{x}_{t}^{i}\right)=0$. Then we argue that $C_{t} \hat{x}_{t}^{i}=C_{0} \hat{x}_{0}^{i}=0$ for $t \geqslant 0$. Finally, we put $C_{t} \hat{x}_{t}^{i}=0$ into (20) to obtain (19).

\section{Conclusion}

Cooperative optical beam tracking, a scheme for maintaining alignment in a free-space optical link, has been addressed. A stochastic model has been developed which captures three sources of randomness: relative motion of stations, characteristic of photodetectors, and fluctuation of optical power caused by optical fade and information-bearing signals modulating the optical beams. An optimal control law has been proposed which maximizes the expected optical energy received by stations of the link. It has been shown that under moderate assumptions, the control law does not depend on the characteristic of the optical fade or information-bearing signals modulating the optical beams.

\section{Appendix A. Proof of Theorem 1}

We prove the first statement of the theorem through the following four steps.

Step I: Recalling that $p_{x_{t}^{i}}\left(x \mid \mathscr{B}_{t}^{i}\right)$ is Gaussian with mean $\hat{x}_{t}^{i}$ and covariance $\Sigma_{t}^{i}$, it is straightforward to show that

$$
\begin{aligned}
\mathrm{E}\left[\exp \left(-\rho\left\|C_{t} x_{t}^{i}\right\|^{2}\right)\right] & =\mathrm{E}\left[\mathrm{E}\left[\exp \left(-\rho\left\|C_{t} x_{t}^{i}\right\|^{2}\right) \mid \mathscr{B}_{t}^{i}\right]\right] \\
& =\mathrm{E}\left[q_{t}^{i} \exp \left(-\rho\left\|Q_{t}^{i} C_{t} \hat{x}_{t}^{i}\right\|^{2}\right)\right]
\end{aligned}
$$

where $Q_{t}^{i}$ and $q_{t}^{i}$ are defined by (14) and (15), respectively. Using the above equation we rewrite (9) as

$$
\begin{array}{r}
J=\mathrm{E}\left[\int_{0}^{T}\left(\alpha^{a} \nu_{t}^{a} q_{t}^{b}+\alpha^{b} \nu_{t}^{b} q_{t}^{a}\right) d t\right] \\
-\mathrm{E}\left[\int_{0}^{T} \alpha^{a} \nu_{t}^{a} q_{t}^{b}\left\{1-\exp \left(-\rho\left\|Q_{t}^{b} C_{t} \hat{x}_{t}^{b}\right\|^{2}\right)\right\} d t\right] \\
-\mathrm{E}\left[\int_{0}^{T} \alpha^{b} \nu_{t}^{b} q_{t}^{a}\left\{1-\exp \left(-\rho\left\|Q_{t}^{a} C_{t} \hat{x}_{t}^{a}\right\|^{2}\right)\right\} d t\right]
\end{array}
$$

Step II: Let $(i, j)=(a, b),(b, a)$. For any $t \geqslant 0$ and for any small positive $\epsilon, \Delta N_{t}^{i} \triangleq N_{t+\epsilon}^{i}-N_{t}^{i}$ is a Poisson random variable conditioned on the rate

$$
\Lambda_{t}^{i}=\int_{t}^{t+\epsilon} \nu_{\tau}^{i} \exp \left(-\rho\left\|C_{\tau} x_{\tau}^{j}\right\|^{2}\right) d \tau
$$


Thus, using the law of total probability we can write

$$
\begin{aligned}
\operatorname{Pr}\left\{\Delta N_{t}^{i}=1 \mid \mathscr{B}_{t}^{j}\right\} & =\mathrm{E}\left[\operatorname{Pr}\left\{\Delta N_{t}^{i}=1 \mid \Lambda_{t}^{i}\right\} \mid \mathscr{B}_{t}^{j}\right] \\
& =\mathrm{E}\left[\Lambda_{t}^{i} e^{-\Lambda_{t}^{i}} \mid \mathscr{B}_{t}^{j}\right] \\
& =p_{t, \epsilon}^{i}+O\left(\epsilon^{2}\right)
\end{aligned}
$$

where $p_{t, \epsilon}^{i}$ is defined as

$$
p_{t, \epsilon}^{i}=\int_{t}^{t+\epsilon} \nu_{\tau}^{i} q_{\tau}^{j} \exp \left(-\rho\left\|Q_{\tau}^{j} C_{\tau} x_{\tau}^{j}\right\|^{2}\right) d \tau
$$

In a similar manner, we can show that

$$
\begin{aligned}
& \operatorname{Pr}\left\{\Delta N_{t}^{i}=0 \mid \mathscr{B}_{t}^{j}\right\}=1-p_{t, \epsilon}^{i}+O\left(\epsilon^{2}\right) \\
& \operatorname{Pr}\left\{\Delta N_{t}^{i} \geqslant 2 \mid \mathscr{B}_{t}^{j}\right\}=O\left(\epsilon^{2}\right)
\end{aligned}
$$

Step III: Let $f(\Sigma)$ be a scalar function of $n \times n$ symmetric matrix $\Sigma$ and assume $f$ is differentiable with respect to $\Sigma$. We can write

$$
\begin{aligned}
\mathrm{E}\left[f\left(\Sigma_{t+\epsilon}^{i}\right)\right] & =\mathrm{E}\left[\mathrm{E}\left[f\left(\Sigma_{t+\epsilon}^{i}\right) \mid \mathscr{B}_{t}^{j}\right]\right] \\
& =\mathrm{E}\left[\sum_{k=0}^{\infty} \mathrm{E}\left[f\left(\Sigma_{t+\epsilon}^{i}\right) \mid \Delta N_{t}^{i}=k, \mathscr{B}_{t}^{j}\right] \operatorname{Pr}\left\{\Delta N_{t}^{i}=k \mid \mathscr{B}_{t}^{j}\right\}\right] \\
& =\mathrm{E}\left[\sum_{k=0}^{1} \mathrm{E}\left[f\left(\Sigma_{t+\epsilon}^{i}\right) \mid \Delta N_{t}^{i}=k, \mathscr{B}_{t}^{j}\right] \operatorname{Pr}\left\{\Delta N_{t}^{i}=k \mid \mathscr{B}_{t}^{j}\right\}\right]+O\left(\epsilon^{2}\right)
\end{aligned}
$$

Also we have

$$
\begin{aligned}
\mathrm{E}\left[f\left(\Sigma_{t+\epsilon}^{i}\right) \mid \Delta N_{t}^{i}=k, \mathscr{B}_{t}^{j}\right] & =\mathrm{E}\left[\mathrm{E}\left[f\left(\Sigma_{t+\epsilon}^{i}\right) \mid \Sigma_{t}^{i}, \Delta N_{t}^{i}=k, \mathscr{B}_{t}^{j}\right] \mid k, \mathscr{B}_{t}^{j}\right] \\
& =\mathrm{E}\left[\mathrm{E}\left[f\left(\Sigma_{t+\epsilon}^{i}\right) \mid \Sigma_{t}^{i}, \Delta N_{t}^{i}=k\right] \mid k, \mathscr{B}_{t}^{j}\right]
\end{aligned}
$$

Inserting the last equation into (25), after some manipulation we find

$$
\mathrm{E}\left[f\left(\Sigma_{t+\epsilon}^{i}\right)\right]=\mathrm{E}\left[\sum_{k=0}^{1} \mathrm{E}\left[f\left(\Sigma_{t+\epsilon}^{i}\right) \mid \Sigma_{t}^{i}, \Delta N_{t}^{i}=k\right] \operatorname{Pr}\left\{\Delta N_{t}^{i}=k \mid \mathscr{B}_{t}^{j}\right\}\right]+O\left(\epsilon^{2}\right)
$$

Conditioned on $\Sigma_{t}^{i}$ and $\Delta N_{t}^{i}=0,1$, the stochastic differential equation (11) can be solved for $\Sigma_{t+\epsilon}^{i}$. This solution leads to

$$
\begin{aligned}
& \mathrm{E}\left[f\left(\Sigma_{t+\epsilon}^{i}\right) \mid \Sigma_{t}^{i}, \Delta N_{t}^{i}=0\right]=f\left(\Sigma_{t}^{i}+\epsilon A_{t} \Sigma_{t}^{i}+\epsilon \Sigma_{t}^{i} A_{t}^{T}+\epsilon D_{t} D_{t}^{T}+O\left(\epsilon^{2}\right)\right) \\
& \mathrm{E}\left[f\left(\Sigma_{t+\epsilon}^{i}\right) \mid \Sigma_{t}^{i}, \Delta N_{t}^{i}=1\right]=f\left(\Sigma_{t}^{i}-M_{t}^{i} C_{t} \Sigma_{t}^{i}+O(\epsilon)\right)
\end{aligned}
$$


We linearize the above equations with respect to $\epsilon$ and plug the linearized equations together with (22) and (24) into (26) to obtain

$$
\begin{aligned}
\mathrm{E}\left[f\left(\Sigma_{t+\epsilon}^{i}\right)\right] & =\mathrm{E}\left[f\left(\Sigma_{t}^{i}\right)+\epsilon \operatorname{tr}\left\{\left(\partial f\left(\Sigma_{t}^{i}\right) / \partial \Sigma_{t}^{i}\right)\left(A_{t} \Sigma_{t}^{i}+\Sigma_{t}^{i} A_{t}^{T}+D_{t} D_{t}^{T}\right)\right\}\right. \\
& \left.+p_{t, \epsilon}^{i}\left(f\left(\Sigma_{t}^{i}-M_{t}^{i} C_{t} \Sigma_{t}^{i}\right)-f\left(\Sigma_{t}^{i}\right)\right)\right]+O\left(\epsilon^{2}\right)
\end{aligned}
$$

Let $g_{t}\left(\Sigma^{a}, \Sigma^{b}\right)$ be a scalar function of $n \times n$ symmetric matrices $\Sigma^{a}$ and $\Sigma^{b}$ and assume its partial derivatives with respect to $t, \Sigma^{a}$ and $\Sigma^{b}$ exist. Then we can write

$$
\mathrm{E}\left[g_{t+\epsilon}\left(\Sigma_{t+\epsilon}^{a}, \Sigma_{t+\epsilon}^{b}\right)\right]=\mathrm{E}\left[g_{t}\left(\Sigma_{t+\epsilon}^{a}, \Sigma_{t+\epsilon}^{b}\right)+\epsilon \partial g_{t}\left(\Sigma_{t+\epsilon}^{a}, \Sigma_{t+\epsilon}^{b}\right) / \partial t\right]+O\left(\epsilon^{2}\right)
$$

Applying (27) to $g_{t}$ and $\partial g_{t} / \partial t$ first for $i=a$ and then for $i=b$, we find

$$
\begin{aligned}
\mathrm{E}\left[g_{t+\epsilon}\left(\Sigma_{t+\epsilon}^{a}, \Sigma_{t+\epsilon}^{b}\right)\right] & =\mathrm{E}\left[g_{t}\left(\Sigma_{t}^{a}, \Sigma_{t}^{b}\right)+\epsilon \partial g_{t}\left(\Sigma_{t}^{a}, \Sigma_{t}^{b}\right) / \partial t\right. \\
& +\epsilon \operatorname{tr}\left\{\left(\partial g_{t}\left(\Sigma_{t}^{a}, \Sigma_{t}^{b}\right) / \partial \Sigma_{t}^{a}\right)\left(A_{t} \Sigma_{t}^{a}+\Sigma_{t}^{a} A_{t}^{T}+D_{t} D_{t}^{T}\right)\right\} \\
& +\epsilon \operatorname{tr}\left\{\left(\partial g_{t}\left(\Sigma_{t}^{a}, \Sigma_{t}^{b}\right) / \partial \Sigma_{t}^{b}\right)\left(A_{t} \Sigma_{t}^{b}+\Sigma_{t}^{b} A_{t}^{T}+D_{t} D_{t}^{T}\right)\right\} \\
& +p_{t, \epsilon}^{a}\left(g_{t}\left(\Sigma_{t}^{a}-M_{t}^{a} C_{t} \Sigma_{t}^{a}, \Sigma_{t}^{b}\right)-g_{t}\left(\Sigma_{t}^{a}, \Sigma_{t}^{b}\right)\right) \\
& \left.+p_{t, \epsilon}^{b}\left(g_{t}\left(\Sigma_{t}^{a}, \Sigma_{t}^{b}-M_{t}^{b} C_{t} \Sigma_{t}^{b}\right)-g_{t}\left(\Sigma_{t}^{a}, \Sigma_{t}^{b}\right)\right)\right]+O\left(\epsilon^{2}\right)
\end{aligned}
$$

In this equation, the term involving $p_{t, \epsilon}^{a}$ can be simplified as

$$
\begin{aligned}
\mathrm{E}\left[p_{t, \epsilon}^{a}\right. & \left.\left(g_{t}\left(\Sigma_{t}^{a}-M_{t}^{a} C_{t} \Sigma_{t}^{a}, \Sigma_{t}^{b}\right)-g_{t}\left(\Sigma_{t}^{a}, \Sigma_{t}^{b}\right)\right)\right] \\
= & \mathrm{E}\left[\int_{t}^{t+\epsilon} \nu_{\tau}^{a} q_{\tau}^{b} \exp \left(-\rho\left\|Q_{\tau}^{b} C_{\tau} x_{\tau}^{b}\right\|^{2}\right) d \tau \cdot \mathcal{L}_{t}^{a}\left\{g_{t}\left(\Sigma_{t}^{a}, \Sigma_{t}^{b}\right)\right\}\right] \\
= & \mathrm{E}\left[\epsilon \nu_{t}^{a} q_{t}^{b} \exp \left(-\rho\left\|Q_{t}^{b} C_{t} x_{t}^{b}\right\|^{2}\right) \mathcal{L}_{t}^{a}\left\{g_{t}\left(\Sigma_{t}^{a}, \Sigma_{t}^{b}\right)\right\}\right]+O\left(\epsilon^{2}\right)
\end{aligned}
$$

Here, the first equation is obtained from (12) and (23) and the second one is concluded from the fact that with probability $1-O(\epsilon)$ the integrand in the first equation is continuous. Applying this result to (28) and rearranging the equation we obtain

$$
\begin{aligned}
& \mathrm{E}\left[g_{t+\epsilon}\left(\Sigma_{t+\epsilon}^{a}, \Sigma_{t+\epsilon}^{b}\right)+\epsilon\left(\alpha^{a} \nu_{t}^{a} q_{t}^{b}+\alpha^{b} \nu_{t}^{b} q_{t}^{a}\right)\right]-\mathrm{E}\left[g_{t}\left(\Sigma_{t}^{a}, \Sigma_{t}^{b}\right)-\epsilon \Gamma_{t}\right]+O\left(\epsilon^{2}\right) \\
& =\epsilon \mathrm{E}\left[\frac{\partial g_{t}\left(\Sigma_{t}^{a}, \Sigma_{t}^{b}\right)}{\partial t}+\nu_{t}^{a} \frac{\alpha^{a}+\mathcal{L}_{t}^{a}\left\{g_{t}\left(\Sigma_{t}^{a}, \Sigma_{t}^{b}\right)\right\}}{\sqrt{\operatorname{det}\left(I+2 \rho C_{t} \Sigma_{t}^{b} C_{t}^{T}\right)}}+\nu_{t}^{b} \frac{\alpha^{b}+\mathcal{L}_{t}^{b}\left\{g_{t}\left(\Sigma_{t}^{a}, \Sigma_{t}^{b}\right)\right\}}{\sqrt{\operatorname{det}\left(I+2 \rho C_{t} \Sigma_{t}^{a} C_{t}^{T}\right)}}\right. \\
& +\operatorname{tr}\left\{\frac{\partial g_{t}\left(\Sigma_{t}^{a}, \Sigma_{t}^{b}\right)}{\partial \Sigma_{t}^{a}}\left(A_{t} \Sigma_{t}^{a}+\Sigma_{t}^{a} A_{t}^{T}+D_{t} D_{t}^{T}\right)\right\} \\
& \left.+\operatorname{tr}\left\{\frac{\partial g_{t}\left(\Sigma_{t}^{a}, \Sigma_{t}^{b}\right)}{\partial \Sigma_{t}^{b}}\left(A_{t} \Sigma_{t}^{b}+\Sigma_{t}^{b} A_{t}^{T}+D_{t} D_{t}^{T}\right)\right\}\right]
\end{aligned}
$$


where $\Gamma_{t}$ is defined as

$$
\begin{aligned}
\Gamma_{t} & =\nu_{t}^{a} q_{t}^{b} \mathcal{L}_{t}^{a}\left\{g_{t}\left(\Sigma_{t}^{a}, \Sigma_{t}^{b}\right)\right\}\left\{1-\exp \left(-\rho\left\|Q_{t}^{b} C_{t} \hat{x}_{t}^{b}\right\|^{2}\right)\right\} \\
& +\nu_{t}^{b} q_{t}^{a} \mathcal{L}_{t}^{b}\left\{g_{t}\left(\Sigma_{t}^{a}, \Sigma_{t}^{b}\right)\right\}\left\{1-\exp \left(-\rho\left\|Q_{t}^{a} C_{t} \hat{x}_{t}^{a}\right\|^{2}\right)\right\}
\end{aligned}
$$

Assuming that $g_{t}(\cdot, \cdot)$ is the backward solution to (16) with boundary condition $g_{T}(\cdot, \cdot)=0$, the right hand side of (29) is identically zero, which yields to

$$
\mathrm{E}\left[g_{t+\epsilon}\left(\Sigma_{t+\epsilon}^{a}, \Sigma_{t+\epsilon}^{b}\right)+\epsilon\left(\alpha^{a} \nu_{t}^{a} q_{t}^{b}+\alpha^{b} \nu_{t}^{b} q_{t}^{a}\right)\right]=\mathrm{E}\left[g_{t}\left(\Sigma_{t}^{a}, \Sigma_{t}^{b}\right)-\epsilon \Gamma_{t}\right]+O\left(\epsilon^{2}\right)
$$

Step IV: Let us denote the first term in the right hand side of (21) by $\tilde{J}$. We partition the interval $[0, T)$ into $K$ subintervals $\left[t_{k}, t_{k+1}\right), k=0,1, \ldots, K-1$, where $t_{0}=0, t_{K}=T$, and $t_{k+1}-t_{k} \triangleq \epsilon_{k}>0$. Then, $\tilde{J}$ can be approximated by

$$
\tilde{J} \simeq \tilde{J}_{K}=\sum_{k=0}^{K-1} \epsilon_{k} \mathrm{E}\left[\alpha^{a} \nu_{t_{k}}^{a} q_{t_{k}}^{b}+\alpha^{b} \nu_{t_{k}}^{b} q_{t_{k}}^{a}\right]+\mathrm{E}\left[g_{t_{K}}\left(\Sigma_{t_{K}}^{a}, \Sigma_{t_{K}}^{b}\right)\right]
$$

Note that $g_{t_{K}}(\cdot, \cdot)=g_{T}(\cdot, \cdot)=0$, thus the last term does not affect the sum and is arbitrarily added to the right hand side. The above equation can be rewritten as

$$
\begin{aligned}
\tilde{J}_{K} & =\sum_{k=0}^{K-2} \epsilon_{k} \mathrm{E}\left[\alpha^{a} \nu_{t_{k}}^{a} q_{t_{k}}^{b}+\alpha^{b} \nu_{t_{k}}^{b} q_{t_{k}}^{a}\right] \\
& +\mathrm{E}\left[g_{t_{K}}\left(\Sigma_{t_{K}}^{a}, \Sigma_{t_{K}}^{b}\right)+\epsilon_{K-1}\left(\alpha^{a} \nu_{t_{K-1}}^{a} q_{t_{K-1}}^{b}+\alpha^{b} \nu_{t_{K-1}}^{b} q_{t_{K-1}}^{a}\right)\right]
\end{aligned}
$$

Since $t_{K}=t_{K-1}+\epsilon_{K-1}$, we can apply (31) to the second term of the right hand side of this equation to obtain

$$
\begin{aligned}
\tilde{J}_{K} & =\sum_{k=0}^{K-2} \epsilon_{k} \mathrm{E}\left[\alpha^{a} \nu_{t_{k}}^{a} q_{t_{k}}^{b}+\alpha^{b} \nu_{t_{k}}^{b} q_{t_{k}}^{a}\right] \\
& +\mathrm{E}\left[g_{t_{K-1}}\left(\Sigma_{t_{K-1}}^{a}, \Sigma_{t_{K-1}}^{b}\right)\right]-\epsilon_{K-1} \mathrm{E}\left[\Gamma_{t_{K-1}}\right]+O\left(\epsilon_{K-1}^{2}\right)
\end{aligned}
$$

Continuing this procedure for $k=K-2, K-3, \ldots, 0$, we obtain

$$
\tilde{J}_{K}=\mathrm{E}\left[g_{t_{0}}\left(\Sigma_{t_{0}}^{a}, \Sigma_{t_{0}}^{b}\right)\right]-\sum_{k=0}^{K-1} \epsilon_{k} \mathrm{E}\left[\Gamma_{t_{k}}\right]+\sum_{k=0}^{K-1} O\left(\epsilon_{k}^{2}\right)
$$

Now we let $K \rightarrow \infty$ and $\max \epsilon_{k} \rightarrow 0$ to obtain

$$
\tilde{J}=\lim _{\substack{N \rightarrow \infty \\ \max \epsilon_{k} \rightarrow 0}} \tilde{J}_{K}=g_{0}\left(\Sigma_{0}^{a}, \Sigma_{0}^{b}\right)-\mathrm{E}\left[\int_{0}^{T} \Gamma_{t} d t\right]
$$

Finally, we put this equation into (21) to obtain (17).

In order to prove the second statement of the theorem, we need the following preliminaries:

P-1) In the context of this proof, we say $f(\cdot): \mathbb{R}^{n \times n} \rightarrow \mathbb{R}$ is strictly decreasing if for any symmetric positive definite matrices $\Sigma$ and $\Delta$, we have $f(\Sigma+\Delta)<f(\Sigma)$. Also we say $f(\cdot)$ is m-positive if for any symmetric positive definite matrix $\Sigma$ we have $f(\Sigma)>0$. 
P-2) If $f_{1}(\cdot)$ and $f_{2}(\cdot)$ are strictly decreasing and m-positive, $f_{1}(\cdot)+f_{2}(\cdot)$ and $f_{1}(\cdot) f_{2}(\cdot)$ are strictly decreasing and m-positive as well.

P-3) If $f(\cdot)$ is strictly decreasing, for any $t$ in which $C_{t}$ is full rank and any positive definite $\Sigma$ we have $f(\Sigma)<f\left(S_{t}(\Sigma)\right)$, where $S_{t}(\cdot)$ is defined by (13).

Proof. Applying the matrix inversion lemma to (13), it is easy to verify that $S_{t}(\Sigma)$ is positive definite for any positive definite $\Sigma$. Also we know from (13) that when $C_{t}$ is full rank, $\Delta \triangleq \Sigma-S_{t}(\Sigma)$ is a positive definite matrix. Because $f(\cdot)$ is assumed to be strictly decreasing, we can write $f(\Sigma)=f\left(S_{t}(\Sigma)+\Delta\right)<f\left(S_{t}(\Sigma)\right)$.

P-4) If $f(\cdot)$ is strictly decreasing and m-positive, for any fixed $t, f\left(S_{t}(\cdot)\right)$ is strictly decreasing and m-positive.

Proof. It is easy to verify that

$$
\Sigma^{-1}-(\Sigma+\Delta)^{-1}=\left(\Sigma+\Sigma \Delta^{-1} \Sigma\right)^{-1}
$$

holds for any invertible matrices $\Sigma$ and $\Delta$. Let $\Sigma$ and $\Delta$ be positive definite matrices. Then (32) implies that $\tilde{\Delta} \triangleq \Sigma^{-1}-(\Sigma+\Delta)^{-1}$ is a positive definite matrix. Using the matrix inversion lemma and replacing $\Sigma^{-1}$ with $(\Sigma+\Delta)^{-1}+\tilde{\Delta}$, we can write

$S_{t}(\Sigma+\Delta)-S_{t}(\Sigma)$

$$
\begin{aligned}
& =\left((\Sigma+\Delta)^{-1}+C_{t}^{T} R_{t}^{-1} C_{t}\right)^{-1}-\left(\Sigma^{-1}+C_{t}^{T} R_{t}^{-1} C_{t}\right)^{-1} \\
& =\left((\Sigma+\Delta)^{-1}+C_{t}^{T} R_{t}^{-1} C_{t}\right)^{-1}-\left(\left((\Sigma+\Delta)^{-1}+C_{t}^{T} R_{t}^{-1} C_{t}\right)+\tilde{\Delta}\right)^{-1}
\end{aligned}
$$

Applying identity (32) to the last equation, we find that $S_{t}(\Sigma+\Delta)-S_{t}(\Sigma)$ is positive definite. Then, because $S_{t}(\Sigma)$ is positive definite and $f(\cdot)$ is strictly decreasing, we have

$$
f\left(S_{t}(\Sigma+\Delta)\right)=f\left(S_{t}(\Sigma)+\left\{S_{t}(\Sigma+\Delta)-S_{t}(\Sigma)\right\}\right)<f\left(S_{t}(\Sigma)\right)
$$

which means $f\left(S_{t}(\cdot)\right)$ is strictly decreasing. Moreover, because $f(\cdot)$ is m-positive and $S_{t}(\Sigma)$ is positive definite, $f\left(S_{t}(\cdot)\right)$ is m-positive.

P-5) For any fixed $t$ in which $C_{t}$ is full rank, $h_{t}(\Sigma)$ defined as

$$
h_{t}(\Sigma)=\frac{1}{\sqrt{\operatorname{det}\left(I+2 \rho C_{t} \Sigma C_{t}^{T}\right)}}
$$

is strictly decreasing and m-positive.

Proof. For positive definite $\Sigma$ and $\Delta$ we can write

$$
\begin{aligned}
\frac{h_{t}(\Sigma)}{h_{t}(\Sigma+\Delta)} & =\sqrt{\frac{\operatorname{det}\left(I+2 \rho C_{t} \Sigma C_{t}^{T}+2 \rho C_{t} \Delta C_{t}^{T}\right)}{\operatorname{det}\left(I+2 \rho C_{t} \Sigma C_{t}^{T}\right)}} \\
& =\sqrt{\operatorname{det}\left(I+2 \rho \Delta^{*}\right)}
\end{aligned}
$$

where $\Delta^{*}$ is defined as

$$
\Delta^{*}=\left(\left(I+2 \rho C_{t} \Sigma C_{t}^{T}\right)^{-1 / 2} C_{t}\right) \Delta\left(\left(I+2 \rho C_{t} \Sigma C_{t}^{T}\right)^{-1 / 2} C_{t}\right)^{T}
$$


Because $\Delta$ is positive definite and $C_{t}$ is full rank, $\Delta^{*}$ is positive definite. This implies that $\operatorname{det}\left(I+2 \rho \Delta^{*}\right)>1$, which leads to $h_{t}(\Sigma+\Delta)<h_{t}(\Sigma)$.

P-6) Let $f_{t}\left(\Sigma^{a}, \Sigma^{b}\right)$ be a scalar function of $n \times n$ matrices $\Sigma^{a}$ and $\Sigma^{b}$. Assume that the function is strictly decreasing and m-positive in both $\Sigma^{a}$ and $\Sigma^{b}$. For $\epsilon>0$ define the linear operator $\mathcal{K}_{t}^{\epsilon}$ as

$$
\mathcal{K}_{t}^{\epsilon} f_{t}\left(\Sigma^{a}, \Sigma^{b}\right)=\left(1-\epsilon \nu_{t}^{a} h_{t}\left(\Sigma^{b}\right)-\epsilon \nu_{t}^{b} h_{t}\left(\Sigma^{a}\right)\right) f_{t}\left(X_{t}^{\epsilon}\left(\Sigma^{a}\right), X_{t}^{\epsilon}\left(\Sigma^{b}\right)\right)
$$

where

$$
X_{t}^{\epsilon}(\Sigma)=\Sigma+\epsilon\left(A_{t} \Sigma+\Sigma A_{t}^{T}+D_{t} D_{t}^{T}\right)
$$

Then, for any symmetric positive definite matrices $\Sigma^{a}, \Sigma^{b}, \Delta^{a}$, and $\Delta^{b}$, there exists $\zeta=\zeta\left(\Sigma^{a}, \Sigma^{b}, \Delta^{a}, \Delta^{b}\right)>0$ such that for any $0 \leqslant \epsilon<\zeta$ we have

$$
\begin{aligned}
\mathcal{K}_{t}^{\epsilon} f_{t}\left(\Sigma^{a}+\Delta^{a}, \Sigma^{b}\right) & <\mathcal{K}_{t}^{\epsilon} f_{t}\left(\Sigma^{a}, \Sigma^{b}\right) \\
\mathcal{K}_{t}^{\epsilon} f_{t}\left(\Sigma^{a}, \Sigma^{b}+\Delta^{b}\right) & <\mathcal{K}_{t}^{\epsilon} f_{t}\left(\Sigma^{a}, \Sigma^{b}\right) \\
\mathcal{K}_{t}^{\epsilon} f_{t}\left(\Sigma^{a}, \Sigma^{b}\right) & >0
\end{aligned}
$$

Therefore, as $\epsilon \rightarrow 0^{+}$, the above condition is satisfied for any choice of $\Sigma^{a}, \Sigma^{b}$, $\Delta^{a}$, and $\Delta^{b}$. This means that if $\epsilon$ lays in a neighborhood of 0 , however small, $\mathcal{K}_{t}^{\epsilon} f_{t}\left(\Sigma^{a}, \Sigma^{b}\right)$ is strictly decreasing and m-positive in both $\Sigma^{a}$ and $\Sigma^{b}$.

We claim that $g_{t}\left(\Sigma^{a}, \Sigma^{b}\right)$, the solution of (16) with boundary condition $g_{T}(\cdot, \cdot)=0$, is strictly decreasing in both $\Sigma^{a}$ and $\Sigma^{b}$ for any $t \in[0, T)$, provided that $C_{t}$ is full rank for $t \in$ $[0, T)$. Once the claim is proven, we apply (P-3) to (12) in order to show $\mathcal{L}_{t}^{i}\left\{g_{t}\left(\Sigma^{a}, \Sigma^{b}\right)\right\}>$ $0, i=a, b$ for any positive definite matrices $\Sigma^{a}$ and $\Sigma^{b}$ and $t \in[0, T)$.

To prove our claim, for any $0 \leqslant t<T$, we partition the interval $[t, T)$ into $K$ subintervals $\left[t_{k+1}, t_{k}\right), k=0,1, \ldots, K-1$, where $t_{K}=t, t_{0}=T$, and $t_{k}-t_{k+1}=\epsilon_{k}>0$. It is straightforward to discretise the partial differential equation (16) over this partition to obtain the recursive equation

$$
\begin{aligned}
g_{t_{k+1}}\left(\Sigma^{a}, \Sigma^{b}\right) & =\epsilon_{k}\left(\alpha^{a} \nu_{t_{k}}^{a} h_{t_{k}}\left(\Sigma^{b}\right)+\alpha^{b} \nu_{t_{k}}^{b} h_{t_{k}}\left(\Sigma^{a}\right)\right) \\
& +\epsilon_{k}\left(\nu_{t_{k}}^{a} h_{t_{k}}\left(\Sigma^{b}\right) g_{t_{k}}\left(S_{t_{k}}\left(\Sigma^{a}\right), \Sigma^{b}\right)+\nu_{t_{k}}^{b} h_{t_{k}}\left(\Sigma^{a}\right) g_{t_{k}}\left(\Sigma^{a}, S_{t_{k}}\left(\Sigma^{b}\right)\right)\right) \\
& +\mathcal{K}_{t_{k}}^{\epsilon_{k}} g_{t_{k}}\left(\Sigma^{a}, \Sigma^{b}\right)+O\left(\epsilon_{k}^{2}\right)
\end{aligned}
$$

Starting from $g_{t_{0}}(\cdot, \cdot)=0$ and using this recursive equation for $k=0,1,2, \ldots K-1$, we can determine $g_{t_{K}}(\cdot, \cdot)$. Then by letting $K \rightarrow \infty$ such that $\max \epsilon_{k} \rightarrow 0$, we have $g_{t_{K}}(\cdot, \cdot) \rightarrow g_{t}(\cdot, \cdot)$.

We prove by induction that as $K \rightarrow \infty$ and $\max \epsilon_{k} \rightarrow 0$, for $k=1,2, \ldots, K, g_{t_{k}}(\cdot, \cdot)$ is strictly decreasing and m-positive in both $\Sigma^{a}$ and $\Sigma^{b}$. From (P-2) and (P-5) we find out that

$$
g_{t_{1}}\left(\Sigma^{a}, \Sigma^{b}\right)=\epsilon_{0}\left(\alpha^{a} \nu_{t_{0}}^{a} h_{t_{0}}\left(\Sigma^{b}\right)+\alpha^{b} \nu_{t_{0}}^{b} h_{t_{0}}\left(\Sigma^{a}\right)\right)
$$

is strictly decreasing and m-positive. Now we show that if $g_{t_{k}}\left(\Sigma^{a}, \Sigma^{b}\right)$ is strictly decreasing and m-positive, $g_{t_{k+1}}\left(\Sigma^{a}, \Sigma^{b}\right)$ is strictly decreasing and m-positive as well. For this purpose, we use $(\mathrm{P}-2, \mathrm{P}-5)$ and $(\mathrm{P}-2, \mathrm{P}-4, \mathrm{P}-5)$ respectively to show that the first and second terms 
on the right hand side of (33) are strictly decreasing and m-positive. Also as $\epsilon_{k} \rightarrow 0^{+}$, (P-6) implies that the third term on the right hand side of (33) is strictly decreasing and $\mathrm{m}$-positive. Because all three terms on the right hand side of (33) are strictly decreasing and m-positive, we conclude from $(\mathrm{P}-2)$ that $g_{t_{k+1}}\left(\Sigma^{a}, \Sigma^{b}\right)$ is strictly decreasing and m-positive.

\section{REFERENCES}

[1] R. M. Gagliardi and S. Karp, Optical Communication, 2nd ed. John Wiley \& Sons, Inc., 1995.

[2] J. M. Lopez and K. Yong, "Laser beam pointing control, acquisition, and tracking," in Laser Satellite Communication, M. Katzman, Ed. Prentice-Hall, Inc., 1987, ch. 6, pp. 190-213.

[3] T. S. Wei and R. M. Gagliardi, "Cooprative optical beam tracking performance analysis," in Acquisition, Tracking, and Pointing II, Proc. SPIE, vol. 887, 1988, pp. 176-183.

[4] G. Marola, D. Santerini, and G. Prati, "Stability analysis of direct-detection cooperative optical beam tracking," IEEE Transactions on Aerospace and Electronic System, vol. 25, no. 3, pp. 325-333, May 1989.

[5] J. W. Goodman, Statistical Optics. John Wiley \& Sons, Inc., 1985.

[6] C. C. Davis., Laser and Electro-Optics Fundamentals and Engineering. Cambridge University Press, 2000.

[7] D. L. Snyder, "Applications of stochastic calculus for point process models arising in optical communication," in Communication Systems and Random Process Theory, J. K. Skwirzynski, Ed., Sijthoff and Noordhoff. Alphen aan der Rijn, The Netherlands, 1978, pp. 789-804.

[8] S. Lee, J. W. Alexander, and M. Jeganathan, "Pointing and tracking subsystem design for optical communications link between the international space station and ground," in Free-Space Laser Communication Technologies XII, Proc. SPIE, S. Mecherle, Ed., vol. 3932, 2000, pp. 150-157.

[9] I. B. Rhodes and D. L. Snyder, "Estimation and control performance for space-time point-process observations," IEEE Transactions on Automatic Control, vol. AC-22, pp. 338-346, Jun. 1977. 